\title{
Classification among Image Enhancement Techniques for Computed Tomography scan by using CancerNet neural network
}

\author{
SatyasangramSahoo ${ }^{\text {a }}$ Dr. R. Lakshmi ${ }^{\mathrm{b}}$
}

Article History: Received: 10 November 2020; Revised 12 January 2021 Accepted: 27 January 2021; Published online: 5 April 2021

\begin{abstract}
Enhancement of cancerous images is a vital section of image preprocessing for Computed Tomography imaging classification. The combination of computer added pictures in X-ray is widely used for medical imaging. Basic enhancement techniques like Pixel wise Enhancements and Local operator based operation on computed Tomography (C.T.) scan are mainly used in preprocessing by using an artificially based model of the medical imaging. The study is focused on selecting the better among basic enhancement methods by using the cancerNet neural network structure. Whereas CancerNet is a widely used Convolutional neural Network structure for classification based study for cancerous medical image.
\end{abstract}

Keywords: Image Pre-processing, Image Enhancement, Pixel-wise Image enhancement, Local operator Image enhancement, Neural Network, CancerNet.

\section{Introduction}

Image enhancement is used to enhance the desired features of the raw image into a better-transformed image. So that either human visual or artificial intelligence-based model can be observed in a better way. This irreversible model can be applied to a single image multiple times for better enhancement. Still, the enhancement's idea is not to lose the objectives of an enhanced image than the original [1]. Sometimes the enhancement of the image may improve noise or unnecessary parts of the image rather than the required portion of an image.

The research primarily focuses on exploiting mixed variants of enhancement processes than single image enhancement in a neural network. The raw image enhancement techniques for medical image processing is the pixel-wise operation and via a local operator. These most-used processing methods are added to the different histogram equalization transformation for a better result [2]. The output images of all these methods are processed with the neural network.

Intensity scaling is an essential aspect of image enhancement. The advanced research on medical image processing is still using the hands-on enhancement method.Gonzalez RC et al. improved medical image performance by using concept intensity scaling[3][4]. The fundamental concept of Histogram equalization is the basis of the theory of intensity scaling. The research is parted the histogram equalization into single-pixel dependent or local Pixel dependent image [5][6].

CancerNet is a neural network architecture used for cancerous image classification [7]. The previous research analysis is proved that the network architecture provides a better result than other models of the convolutional neural network. The CancerNet is not meant only for medical cancerous image classification, but this can ensure more accuracy when it deals with the scan tumor C.T. images' sensitiveness.

\section{Method}

\subsection{Datasets}

Datasets are an essential prospect for any neural network classification approach. Luna16 and LIDC - IDRI datasets for lung cancer are chosen for the research. Different formats like DICOM are converted to Joint Photographic Expert Group (JPEG) for further processing. JPEG format is selected due to its minimization size of an image to process these large datasets.As the idea is for evaluation for better image enhancement methods in preprocessing, so the same Images are first processed and then categorized according to the number of enhancement techniques used for the classification.

LIDC - IDRI dataset is included with an annotation XML file with 1018 CT Scan images[8]. According to annotation, It is categorized into three folders, i.e., nodule $\geq 3 \mathrm{~mm}$, nodule $\leq 3 \mathrm{~mm}$, and non-nodule $\geq 3 \mathrm{~mm}$. The version of the LUNA16 dataset that is used for the paper consists of a total of 888 CT images with two categorizations [9]. The similar making structure for both the dataset in the module, which is less than the $3 \mathrm{~mm}$ 
and non-nodule, is grouped into a single set as "Non - relevant." The other was tagged as "Relevant / Malignant."

\subsection{Pre-processing}

The dataset of only the raw C.T. scan images is used for this research purpose. The primary intention is to process all the image enhancement techniques of the same image in these two primary enhancement methods. The comparison study is for only the preprocessing of scan images, so any pre-modified or enhanced image is not taken for the research.This comparison study shows every image enhancement by using the primary enhancement method by using CancerNet architecture.
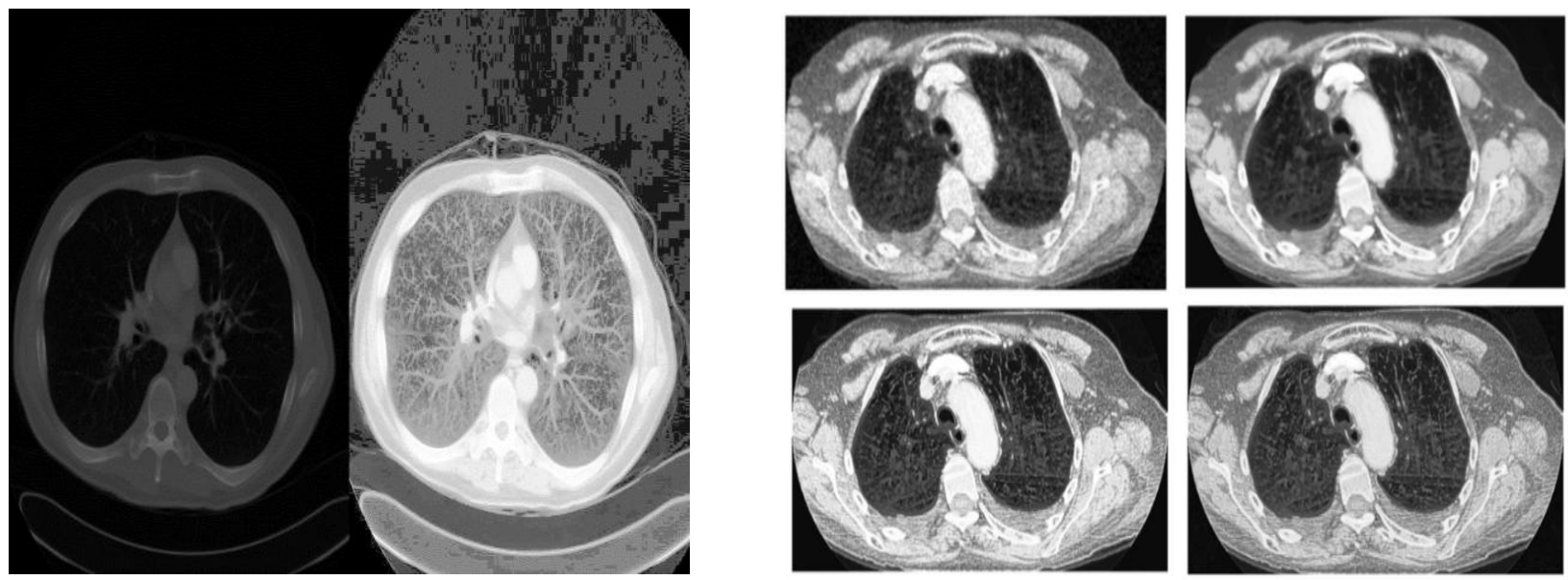

Fig 1: Pixel wise modified C.T. image (Right side) is the enhanced version of the raw image (Left side).Fig 2: Local operator enhancement method a) Raw image of a dataset (top left) b) after applying median filter (top right) c) edge was enhanced on filtered image (bottom left) d) final image after applying local histogram equalization (bottom right)

In the preprocessing section of image enhancement, the downloaded image is varied by different formats and sizes. All the C.T. scans are binarized to their greyscale images, each of size $221 \times 221$. All the data is divided into the Train and test set of the ratio 80:20.These images are needed to be standardized in a specific format. Joint Photographic Expert Group is selected against other usable standards like PNG as it consumes less storage. Due to less size of the JPEG format, this standard is useful for deep neural processing as an efficient and less timeconsuming format.

\subsection{Method}

The fundamental enhancement of an image in preprocessing stage is broadly categorized into two methods. These are pixel-wise enhancement and local operator wise enhancement. The essential enhancement is to add some extra feature to present the image in the advanced enhancement method. But the crucial element is still in use in some manual enhancement, particularly in medical imaging, to get detail in the region of interest of any image.

Pixel operation is defined as enhancing the pixel grey level of an image, which does not depend upon the neighborhood pixels. On the other hand, the Local operator image enhancement, each pixel value in the picture, is enhanced by using Pixel's value along with neighborhood pixels. As both, image enhancementsare popularly used in medical image classification. This research is the comparative study of both the enhancement techniques by using transfer learning with the help of the convolutional neural network.

The histogram of an image is used for image characterization and enhancement techniques. It is a vector of countable numbers of pixels at each grey level of an image. The equation of histogram (h(i)) can be defined as

$$
h(i)=\sum_{x=0}^{X-1 Y-1} \sum_{y=0} \delta(f(x, y)-i)
$$

$\mathrm{i}=0,1,2, \ldots . \mathrm{P}-1$,

Where

$$
\delta(f(x, y)-i)=\left\{\begin{array}{cc}
1, & \text { if } f(x, y)-i=0 \\
0, & \text { otherwise }
\end{array}\right.
$$


Here $(x, y)$ is the position of the Pixel in an image. $f(x, y)$ is the Intensity of that Pixel. $X$ is the maximum number of rows whereas

$\mathrm{Y}$ is the maximum number of the Column of the Image. $\mathrm{P}$ is the quantized grey intensity levels available in the image, which can vary from 0 to $\mathrm{P}-1$.

Intensity scaling is an essential pixel operation image enhancement technique that allows modifying specific narrow band intensity band to span across the dynamic range of the display band[10][11].

Histogram equalization is the most popular method of intensity scaling. In this technique, an image's acquired intensity is uniformly distributed across the whole intensity available band[5]. The image histogram's output is flatter intensity bands than the input[6][12]. The redistribution of each image pixel from the no uniform raw image histogram is

$$
H(j)=\frac{1}{X . Y} \sum_{j=0}^{j} h(i)
$$

Where $\mathrm{j}=0,1, \ldots . \mathrm{P}-1$

The enhanced Image of a uniformly distributed histogram is defined as

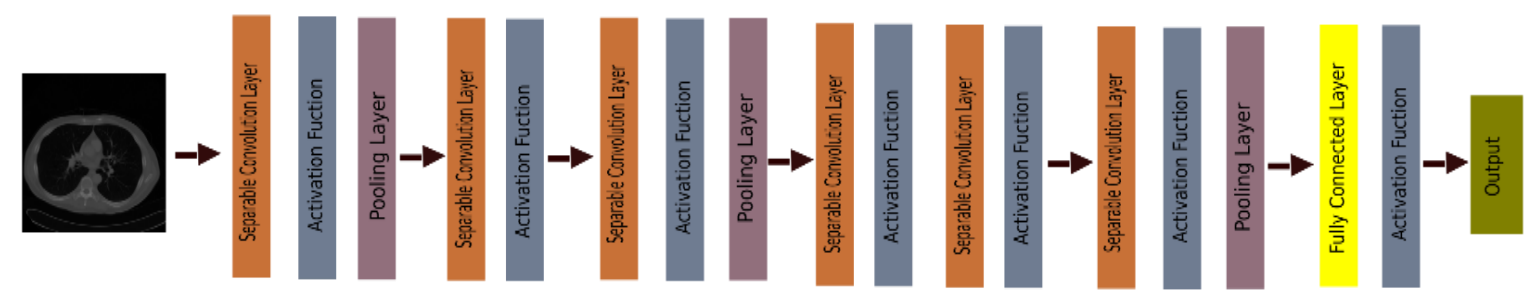

CancerNet Archetecture

$$
\mathrm{g}(\mathrm{x}, \mathrm{y})=(\mathrm{P}-1) \cdot \mathrm{H}(\mathrm{f}(\mathrm{x}, \mathrm{y}))
$$

The local operator is different from pixel-wise operation by adding a dependency of neighboring pixels along with the Pixel to change its value. Noise cancellation with filtering and edge enhancement along with histogram equalization within a neighborhood are the primary methods done by local operator techniques[13].

The Local operator-based operation is a three-step algorithm. Initially, the image is processed, either mean or median filtering. In this experiment, Median based filtering is applied, which is then followed by edge enhancement. Finally, the enhancement stages are ended with Local area histogram equalization.

Median filtering, a standard nonlinear noise cancellation method, is applied to the Lung cancer image for better removal of substantial noise. In the median filter, the kernel frame is convoluted to each Pixel of a lung cancer image. The new pixel value is calculated as the Pixel's median value within the kernel frame. After noise removal, edge enhancement is the essential step to preserving the context and enhancing an image's edge. In the enhancement technique,

The kernel's convolution enhances the image edge after adding value one to its central coefficient [4][13].

The local area histogram equalization is a nonlinear operation where the whole image histogram is equalized to a local portion of an image. This feature has the capability of increasing the observability of subtle parts of an image

The Equation of The Local histogram is

$$
h_{L A}(x, y)(i)=\sum_{k=-K l=-L}^{K} \sum_{l}^{L} \delta(f(x+l, y+k)-i)
$$

The equation of the local cumulative histogram is

$$
H_{L A}(x, y)(j)=\frac{1}{(2 X+1) \cdot(2 Y+1)} \sum_{i=0}^{j} h_{L A}(x, y)(i)
$$

Where $\mathrm{i}$ and $\mathrm{j}=0,1 \ldots \mathrm{P}-1$

The Output Enhanced image is

$$
g(x, y)=(P-1) \cdot H_{L A}(x, y)(f(x, y))
$$


This computationally intensive enhancement method is mapped hidden detail spanned fewer pixels into nonmonotonic and nonlinear higher-visibility pixels of the whole image.

Convolutional Neural Network is used for this classification task. For an image like a C.T. scan of cancer, Depth wise separable convolution will produce more accuracy than standard Convolutional Neural Network architecture. CancerNet is chosen among the depth-wise convolution Neural Network[7]. The architecture has shared similar properties to VGGNet architecture. CancerNet requires less computation time and space for better output.

\section{Result}

The research is a comparison study among the two basic enhancement techniques. The raw image without any further enhancement is processed into the neural network for comparison study. The raw image with CancerNet architecture is shown $89.87 \%$ accuracy with an error rate of $10.13 \%$. In contrast, Pixel operation image enhancement has improved up to $92.26 \%$ accuracy with an error rate of $7.74 \%$. It has improved significantly from the raw image classification task. The local operator, including histogram equalization, is markedly varied from a single pixel-wise enhancement operation. The Local operator is shown an accuracy of $94.02 \%$ with an error rate of $5.98 \%$.

\section{Conclusion}

Image enhancement is a necessary stage for the medical image as a modified version can classify better than raw computed tomography images. The fundamental image enhancement method on medical scan images is taken into account to classify. In this paper, pixel-wise enhancement and local image enhancement techniques are counted for this research. The later technology like Fourier Transformation and other techniques are excluded, which inclusion might have shown different results. As these image enhancement methods are widely used before any segmentation or classification, the exclusion of other vital aspects is necessary to show in this research. Local image enhancement is enhanced with the help of the surrounding Pixel along with that Pixel.

\section{References}

Nixon, Mark, and Alberto Aguado. Feature extraction and image processing for computer vision. Academic Press, 2019.

Wang, Yanfang, Qian Huang, and Jing Hu. "Adaptive Enhancement for non-uniform illumination Images via pixel-wise Histogram Modification and Color Reconstruction." 2018 IEEE 3rd International Conference on Signal and Image Processing (ICSIP). IEEE, 2018.

Gonzalez, Rafael C., and Richard E. Woods. "Digital Image Processing (preview)." (2002).

Gonzalez RC, Woods RE. Digital Image Processing. Reading, MA: Addison-Wesley; 1992

Frei, Werner. "Image enhancement by histogram hyperbolization." Computer Graphics and Image Processing 6.3 (1977): 286-294

Hall, Ernest L. "Almost uniform distributions for computer image enhancement." IEEE Transactions on Computers 100.2 (1974): 207-208.

Adrian Rosebrock, breast-cancer-classification-with-keras-and-deeplearning,https://www.pyimagesearch.com/2019/02/18/breast-cancer-classification-with-keras-and-deeplearning/accessed on 23 april.

Armato Samuel, G., III; McLennan, G.; Bidaut, L.; McNitt-Gray, M.F.; Meyer, C.R.; Reeves, A.P.; Zhao, B.; Aberle, D.R.; Henschke, C.I.; Hoffman, E.A.; et al. Data From LIDC-IDRI. 2015. Available online: https: //wiki.cancerimagingarchive.net/display/Public/LIDC-IDRI (accessed on 7 January 2020)

Setio, A.A.A.; Traverso, A.; de Bel, T.; Berens, M.S.N.; van den Bogaard, C.; Cerello, P.; Chen, H.; Dou, Q.; Fantacci, M.E.; Geurts, B.; et al. Validation, Comparison, and Combination of Algorithms for Automatic Detection of Pulmonary Nodules in Computed Tomography Images: The LUNA16 Challenge.Med. Image Anal. 2017, 42, 1-13.

Russ J. The Image Processing Handbook, 2nd ed. Boca Raton, FL: CRC Press; 1994.

Wahl F. Digital Image Signal Processing. Norwood, MA: Artech House; 1987

Ketcham, David J. "Real-time image enhancement techniques." Image processing. Vol. 74. 1976.

Fong, Yu-Shan, Carlos A. Pomalaza-Raez, and Xiao-Hua Wang. "Comparison study of nonlinear filters in image processing applications." Optical Engineering 28.7 (1989): 287749. 